\title{
Estudo da recuperação do herbicida atrazina em águas naturais empregando Cromatografia Líquida de Alta eficiência (HPLC) \\ Pricília Santos Pereira Gomes ${ }^{1}$; Luciana Bagdeve de Oliveira dos Santos²; Luiza Maria Cerqueira Silva ${ }^{3}$ \\ 1. Bolsista PIBIC/FAPESB, Graduando em Licenciatura em Química, Universidade Estadual de Feira de Santana, e- mail: priciliagomes13@gmail.com \\ 2. Orientador, Departamento de Exatas, Universidade Estadual de Feira de Santana, e-mail: lbagdeve@ @otmail.com \\ 3. Mestranda, Departamento de Química Universidade Federal da Bahia, e-maill: lusilva92@live.com
}

PALAVRAS-CHAVE: Atrazina; HPLC; Águas naturais.

\section{INTRODUÇÃO}

Os pesticidas são empregados, em larga escala, no controle de pragas em vários países, entretanto, estes podem ser nocivos ao meio ambiente, agredindo o solo e a água, além de apresentar fatores de risco a saúde de animais, inclusive o ser humano, haja vista que o índice de intoxicação por pesticidas é alto, principalmente em países em desenvolvimento (Delgado \& Paumgartten, 2004). Dentre os herbicidas mais utilizados no mundo, tem-se a atrazina, pertencente à família das triazinas. Estes herbicidas podem sofrer degradação biológica ou química após serem aplicadas ao solo, formando metabólitos que são muitas vezes mais nocivos que o próprio herbicida. O uso exacerbado pode comprometer a qualidade da água dos rios, lagos e outras reservas de água potável, pois é empregado em vários tipos de culturas

A atrazina (AT) apresenta alta persistência em diferentes ambientes aquáticos, atrelado a isso grande potencial danoso para animais que vivem neste meio e ainda para o ser humano. Existem três principais vias de degradação de AT para formação de metabolitos: dealquilação biológica, hidrólise química e hidrólise biológica (COELHO \& BERNARDO, 2012). Com a degradação de AT, vários metabolitos podem ser formados, dentre estes, 2-hidroxiatrazina (ATOH), desetilatrazina (DEA) e desisopropilatrazina (DIA) e desetildeisopropilatrazina (DEDIA), objetos de estudo deste trabalho. Este trabalho visa desenvolver uma metodologia de separação de AT, ATOH, DIA, DEA e DEDIA empregando Cromatografia Líquida de Alta Eficiência (HPLC) utilizando colunas $\mathrm{C}_{18}$ convencional e monolítica para recuperação desses analitos em amostras de águas naturais.

\section{MATERIAL E MÉTODOS OU METODOLOGIA (ou equivalente) Coleta e caracterização de águas}

Foram coletadas amostras de água das Lagoas do Parque Frei Monteiro e da Pindoba, além de água tratada (Embasa). A coleta foi realizada de acordo com o procedimento de amostras simples (coletada de uma única vez) com coleta superficial (profundidade entre 0 e $30 \mathrm{~cm}$ ) em recipientes como estabelece a NBR 9898 e para água tratada foi aplicado o teste de cavalete.

Otimização do método para determinação do pesticida AT e dos metabólitos DEA, DIA, ATOH e DEDIA

A atrazina e seus metabólitos foram determinados HPLC, utilizando detector UV em comprimento de onda $220 \mathrm{~nm}$ e coluna cromatográfica $\mathrm{C}_{18}$ convencional e monolítica. As condições de fluxo e composição da fase móvel, acetonitrila (ACN) e 
tampão acetato 2,5mmol/L, foram otimizadas para obtenção da melhor condição de análise.

\section{Formação de metabolitos}

As amostras foram contaminadas com $1 \mathrm{~mL}$ de atrazina $50 \mathrm{mg} \mathrm{L}^{-1}$ resultando em solução $0,50 \mathrm{mg} \mathrm{L}^{-1}$ e foram analisadas após 55 dias por HPLC com ambas colunas.

\section{RESULTADOS E/OU DISCUSSÃO (ou Análise e discussão dos resultados) Caracterização físico-químicas das amostras}

Tabela 1. Parâmetros físico-químicos das amostras

\begin{tabular}{l|llllllll}
\hline Amostras & $\boldsymbol{p H}$ & $\begin{array}{l}\text { Condutividade } \\
(\boldsymbol{m} \boldsymbol{S})\end{array}$ & $\begin{array}{l}\text { Resistência } \\
(\mathbf{\Omega})\end{array}$ & $\begin{array}{l}\text { OD } \\
(\mathbf{m g} / \mathbf{L})\end{array}$ & $\begin{array}{l}\boldsymbol{S T D} \\
(\mathbf{p p m})\end{array}$ & $\begin{array}{l}\text { Turbidez } \\
(\mathbf{N T U})\end{array}$ & $\begin{array}{l}\text { Salinidade } \\
(\boldsymbol{p p m})\end{array}$ & $\begin{array}{l}\text { Temperatura } \\
\left({ }^{\circ} \boldsymbol{C}\right)\end{array}$ \\
\hline $\begin{array}{l}\text { Lagoa da } \\
\text { Pindoba }\end{array}$ & 5,64 & 16,67 & 132,2 & 1,13 & 7,765 & 26,4 & $4,255^{*} 10^{-3}$ & 26,9 \\
$\begin{array}{l}\text { Parque } \\
\text { Frei }\end{array}$ & 7,62 & 1,773 & $1.247,0$ & 0,86 & 401,6 & 22,2 & 394,4 & 24,5 \\
$\begin{array}{l}\text { Monteiro } \\
\text { EMBASA }\end{array}$ & 7,01 & 0,2733 & $3.118,0$ & 0,23 & 160,3 & 3,19 & 154,3 & 21,4 \\
\hline
\end{tabular}

Legenda: OD:Oxigênio Dissolvido; STD: Sólidos totais dissolvidos

\section{Separação com coluna $\mathbf{C}_{18}$ convencional}

Diante das análises efetuadas com modo isocrático de eluição, concluímos que era inviável a separação de todos os analitos em uma única analise, nesse sentido, foi necessário utilizar eluição por gradiente de polaridade. No gradiente empregado utilizou-se as condições ótimas de separação para cada um dos analitos, obtidos na eluição isocrática. Iniciou-se na condição 10/90 (ACN/ Tampão acetato 2,5 mmol L-1), na qual temos a separação da DEDIA, alterando em seguida para 35/65 (ACN/Tampão acetato 2,5 mmol L $\mathrm{L}^{-1}$ ), condição na qual temos a separação da ATOH, DIA e DEA e finalizando com 65/35(ACN/Tampão acetato 2,5 $\mathrm{mmol} \mathrm{L}^{-1}$ ) para AT. Os resultados nas condições otimizadas são apresentados na Figura 01.

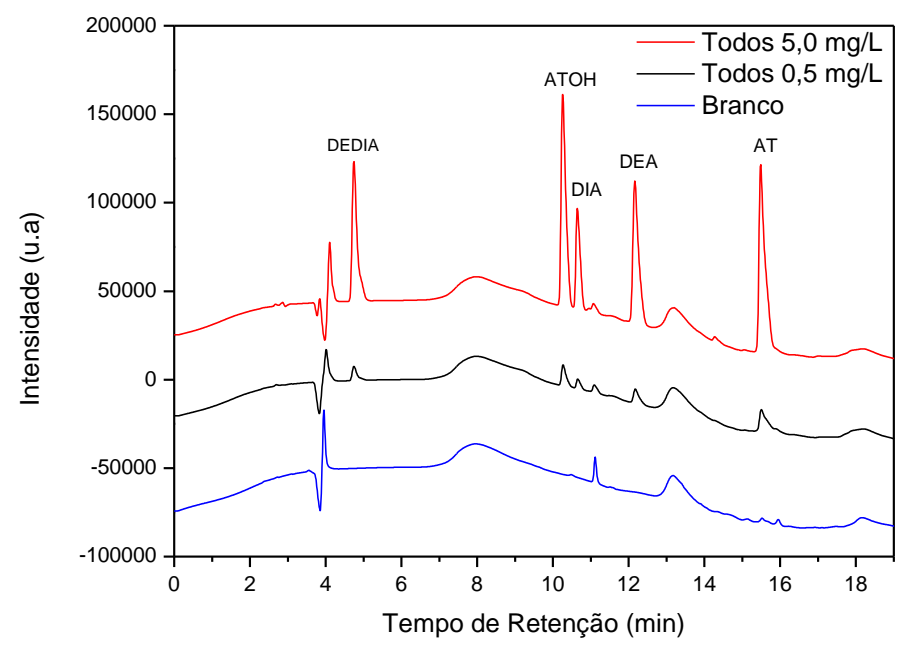

Figura 1: Separação cromatográfica com coluna convencional de AT, DEA, DIA, DEDIA E ATOH em Condição gradiente de fase móvel acetonitrila/tampão acetato $2,5 \mathrm{mmol} \mathrm{L}{ }^{-1}$, Vazão de fase móvel= 1,0 $\mathrm{mL} \min ^{-1}, \mathrm{TC}=40^{\circ} \mathrm{C}$ e branco constituído de tampão acetato $2,5 \mathrm{mmol} \mathrm{L}{ }^{-1}$. 


\section{Separação com coluna $\mathrm{C}_{18}$ monolítica}

Para programação de gradiente com a coluna monolítica, utilizou-se as condições ótimas dos analitos, avaliados individualmente com eluição isocrática. Iniciou-se na condição 3/97 (ACN/ Tampão acetato $2,5 \mathrm{mmol} \mathrm{L}^{-1}$ ), na qual temos a separação da DEDIA, alterando em seguida para 20/80 (ACN/Tampão acetato 2,5 mmol $\mathrm{L}^{-1}$ ), condição na qual temos a separação da ATOH, DIA e DEA e finalizando com 60/40(ACN/Tampão acetato $2,5 \mathrm{mmol} \mathrm{L}^{-1}$ ) para AT. Os resultados nas condições otimizadas são apresentados na Figura 02.

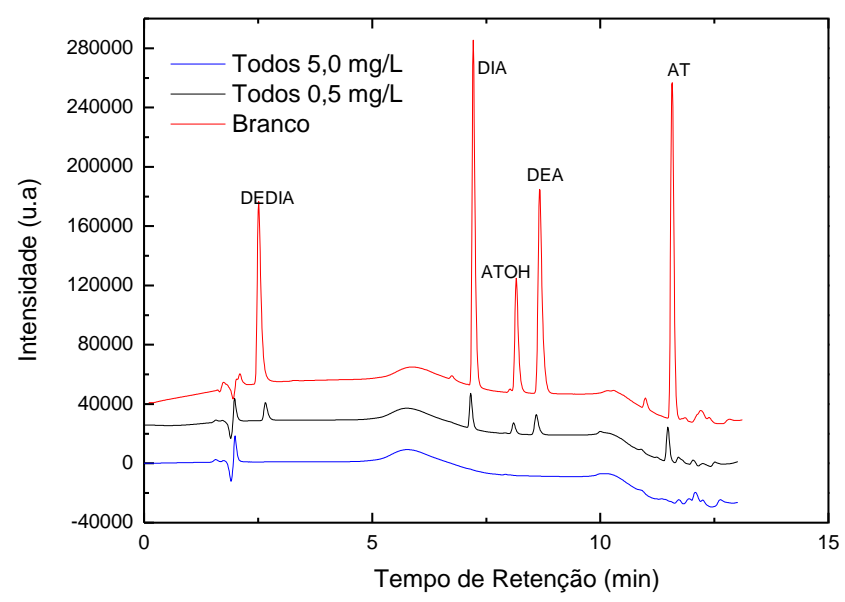

Figura 2: Separação cromatográfica com coluna monolítica de AT, DEA, DIA, DEDIA E ATOH em Condição gradiente de fase móvel acetonitrila/tampão acetato $2,5 \mathrm{mmol} \mathrm{L}{ }^{-1}$, Vazão de fase móvel= 1,0 $\mathrm{mL} \min ^{-1}, \mathrm{TC}=40^{\circ} \mathrm{C}$ e branco constituído de tampão acetato $2,5 \mathrm{mmol} \mathrm{L}{ }^{-1}$.

\section{Parâmetros Analíticos}

Tabela 2. Curvas Analíticas para AT, ATOH, DIA, DEA e DEDIA nas condições otimizadas de gradiente com coluna $\mathrm{C} 18$ convencional e monolítica

\begin{tabular}{|c|c|c|c|c|c|c|}
\hline & \multicolumn{3}{|c|}{ Coluna Convencional } & \multicolumn{3}{|c|}{ Coluna monolítica } \\
\hline & $\mathrm{B}(\mathrm{mg} / \mathrm{L})$ & $\mathrm{R}^{2}$ & $\begin{array}{c}\text { F. Linear } \\
(\mathrm{mg} / \mathrm{L})\end{array}$ & $\mathrm{B}(\mathrm{mg} / \mathrm{L})$ & $\mathrm{R}^{2}$ & $\begin{array}{c}\text { F. Linear } \\
(\mathrm{mg} / \mathrm{L})\end{array}$ \\
\hline DEDIA & $144374 \pm 707$ & 0,9998 & $0,05-25$ & $143897 \pm 720$ & 0,9998 & $0,01-10$ \\
\hline ATOH & $194128 \pm 2274$ & 0,9989 & $0,05-50$ & $83214 \pm 259$ & 0,9999 & $0,01-10$ \\
\hline DIA & $90726 \pm 235$ & 0,9999 & $0,05-50$ & $209626 \pm 616$ & 0,9999 & $0,01-10$ \\
\hline DEA & $160900 \pm 1453$ & 0,9993 & $0,05-50$ & $172041 \pm 460$ & 0,9999 & $0,01-10$ \\
\hline AT & $230766 \pm 1499$ & 0,9997 & $0,025-25$ & $224718 \pm 426$ & 0,9999 & $0,01-10$ \\
\hline
\end{tabular}

Legenda: A equação da reta é dada por y= A + Bx e F. Linear é a Faixa Linear de Trabalho; $y=$ área; $\mathrm{x}=$ concentração; $\mathrm{b}=$ sensibilidade (inclinação da curva); $a=$ interseção com eixo y, quando $\mathrm{x}=0$

As curvas analíticas foram plotadas de acordo as condições de separação nas Figuras 1 e 2. Nota-se como apresentado na Tabela 1 que as sensibilidades para os analitos de DEDIA, AT e DEA com as duas colunas são equivalentes. Em ATOH a maior sensibilidade é obtida com a coluna convencional, enquanto que para DIA é a coluna monolítica. 
Tabela 3. Valores de recuperação de atrazina nas amostras lagoas da Pindoba, do Parque

Frei Monteiro e Embasa após a contaminação com AT 50,0 $\mathrm{mg} \mathrm{L}^{-1}$ resultando em solução $0,50 \mathrm{mg} \mathrm{L}^{-1}$

\begin{tabular}{c|ccccc}
\hline & \multicolumn{4}{|c}{ Coluna C18 Convencional } & \multicolumn{2}{c}{ Coluna C18 Monolítica } & \\
\hline $\begin{array}{c}\text { Nome da } \\
\text { amostra }\end{array}$ & Média $\left(\mathbf{m g ~ L}^{-\mathbf{1}}\right)$ & $\begin{array}{c}\text { Desvio (S) } \\
\left(\mathbf{m g ~ L}^{-1}\right)\end{array}$ & Média $\left(\mathbf{m g ~ L}^{-\mathbf{1}}\right)$ & $\begin{array}{c}\text { Desvio (S) } \\
\left(\mathbf{m g ~ L}^{-1}\right)\end{array}$ & $|\mathbf{t}|$ \\
Pindoba & 0,491 & 0,001 & 0,44 & 0,02 & 2,75 \\
$\begin{array}{c}\text { Parque F. } \\
\text { Monteiro }\end{array}$ & 0,41 & 0,01 & 0,42 & 0,01 & 0,93 \\
Embasa & 0,467 & 0,004 & 0,44 & 0,01 & 2,14 \\
\hline
\end{tabular}

Legenda: Valor de t para 4 graus de liberdade e $95 \%$ de confiança igual a 2,78.

As concentrações de atrazina foram obtidas usando as curvas analíticas de ambas as colunas cromatográficas (Tabela 2). Os resultados obtidos para recuperação de atrazina nas amostras de águas, mostraram recuperação acima de $82 \%$ do pesticida, no entanto, não foi detectado a presença de metabolitos nas determinações realizadas. Os métodos utilizando coluna C18 convencional e C18 monolítica foram comparados empregando o teste $\mathrm{t}$ de Student para $95 \%$ de confiança, e uma vez que os valores de " $\mathrm{t}$ " obtidos foram menores que o " $\mathrm{t}$ " tabelado $=2,78$, podemos afirmar que não existem evidências de diferenças significativas entre os métodos empregados, sendo que as determinações obtidas por coluna monolítica apresentaram menor tempo de análise.

\section{CONSIDERAÇÕES FINAIS (ou Conclusão)}

As condições de eluição que separam atrazina e os metabolitos em uma única analise estudados neste trabalho é por gradiente de fase móvel, tanto para a coluna C18 convencional quanto para C18 monolítica. Condições apresentadas nas Figuras 1 e 2 apresentam boa separação e resolução. Os testes de repetitividade dos métodos empregados demonstram que os coeficientes de variação obtidos foram inferiores a 5\% para ambos os métodos. Os resultados obtidos para recuperação de atrazina nas amostras de águas, mostraram recuperação acima de $82 \%$ do pesticida, no entanto, não foi detectado a presença de metabolitos nas determinações realizadas. Os métodos empregados foram comparados usando teste t de Student para $95 \%$ de confiança, como os valores de " $\mathrm{t}$ " obtidos foram menores que o " $\mathrm{t}$ " tabelado $=2,78$, podemos afirmar que os métodos desenvolvidos são equivalentes, ou seja, serão obtidas respostas de analises satisfatórias utilizando tanto a coluna $\mathrm{C}_{18}$ Convencional quanto a $\mathrm{C}_{18}$ Monolitica. A coluna monolítica apresentou limites de detecção e tempos de retenção menores que a coluna convencional para todos os analitos.

\section{REFERÊNCIAS}

COELHO, Edumar Ramos Cabral; BERNARDO, Luiz Di. Removal of atrazine and metabolites through slow filtration by sand and granular activated carbon. Engenharia Sanitaria e Ambiental, 2012.

DELGADO, Isabella Fernandes; PAUMGARTTEN, Francisco José Roma. Intoxicações e uso de pesticidas por agricultores do Município de Paty do Alferes, Rio de Janeiro, Brasil, 2004. Disponível em:<http://www.scielosp.org/pdf/csp/v20n1/34.pdf>. Acesso em: 14 de fevereiro de 2017. 